\title{
A Report of 2 Cases of Tetra-Amelia Syndrome
}

\section{Abstract}

The objective of this study was to report 2 cases of tetra-amelia syndrome. The first case corresponded to a tetra-amelia-1 syndrome with, in addition, facial and probably pulmonary anomalies. He was born 36 weeks of gestation and died within the first 10 minutes of life. The second case had a tetra-amelia- 2 phenotype with a symmetrical amelia. No other abnormalities were detected on ultrasound. Tetra-amelia syndrome is extremely rare; it is linked to genetic mutations that cause great phenotypic heterogeneity

Keywords: Tetra-amelia; Malformation; Genotype; Phenotype.

Received: January 18, 2020; Accepted: February 04, 2020; Published: February 11, 2020

\section{Mame Diarra $\mathbf{N}^{1 *}$, Mamour G ${ }^{1}$, Simon Birame $\mathbf{N}^{1}$, Abdoulaye $\mathrm{D}^{1}$, Ndeye Gothe FLL², Magatte $M^{2}$ and Jean Charles $\mathbf{M}^{2}$}
1 Gynecologic and Obstetric Clinic, Aristide Le Dantec Teaching Hospital, Pasteur Avenue, Cheikh Anta Diop University, Dakar, Senegal
2 Philippe Senghor Health Center, Cheikh Anta Diop University, Dakar, Senegal

*Corresponding author: Mame Diarra N

\section{Introduction}

Limb anomalies constitute an important group of congenital pathologies characterized by hypoplasia or complete aplasia of one or more limb bones. Limb abnormalities of all types occur in approximately 1 in 1,300 to 2,000 births. These limb anomalies can be isolated or associated with other anomalies [1]. Tetraamelia syndrome is rare and gray areas remain.

We report two cases of tetra-amelia in a level II maternity in Dakar (Senegal) with to be similar to tetraamelia-1 (chromosome 17q21), tetraamelia-2 (chromosome 8q23) and Robert's syndrome (chromosome 8p21). This illustrates the difficulty in correlating phenotype and genes involved.

\section{Case Reports}

\section{Case 1}

Ms. AD was a 44-year-old mother referred to our department at 36 weeks of gestation with severe pre-eclampsia and fetal anomalies. She was five para with no history of fetal anomalies. She did not smoke now and had never neither smoked nor drunk alcohol. She had not been exposed to passive smoking. She was in a third-degree consanguineous marriage for all her children. Ms. $A D$ had tested negative for hepatitis B, HIV and syphilis. She was protected from the rubella virus and had no previous exposure to Toxoplasma gondii. Ultrasound monitoring performed late at 33 weeks and 35 weeks of gestation retrieved oligoamniosis and hydrocephalus as well as agenesis of the limbs. Prescriptions during pregnancy included the administration of iron and folic acid, as well as the administration of sulfadoxine pyrimethamine. The latter was prescribed at 18 weeks and then 26 weeks as part of the anti-malaria prophylaxis policy for pregnant women. The symphyseal-fundal height measured $28 \mathrm{~cm}$. Due to severe features of pre-eclampsia; she was hospitalized immediately and observed in a labor and delivery unit. She then initially received IV magnesium sulfate to prevent eclampsia and antihypertensive medications to maintain systolic blood pressure below $160 \mathrm{mmHg}$ and diastolic blood pressure below $105 \mathrm{mmHg}$.

The decision of immediate caesarean delivery was made. A 2,150 gram live-born was extracted which subsequently died within 10 minutes. Before death, the body presented crawling movements. Several external anomalies were identified (Figure 1) including complete agenesis of all four limbs, hydrocephalus with a head circumference of $39 \mathrm{~cm}$. On the face, there was hypertelorism 

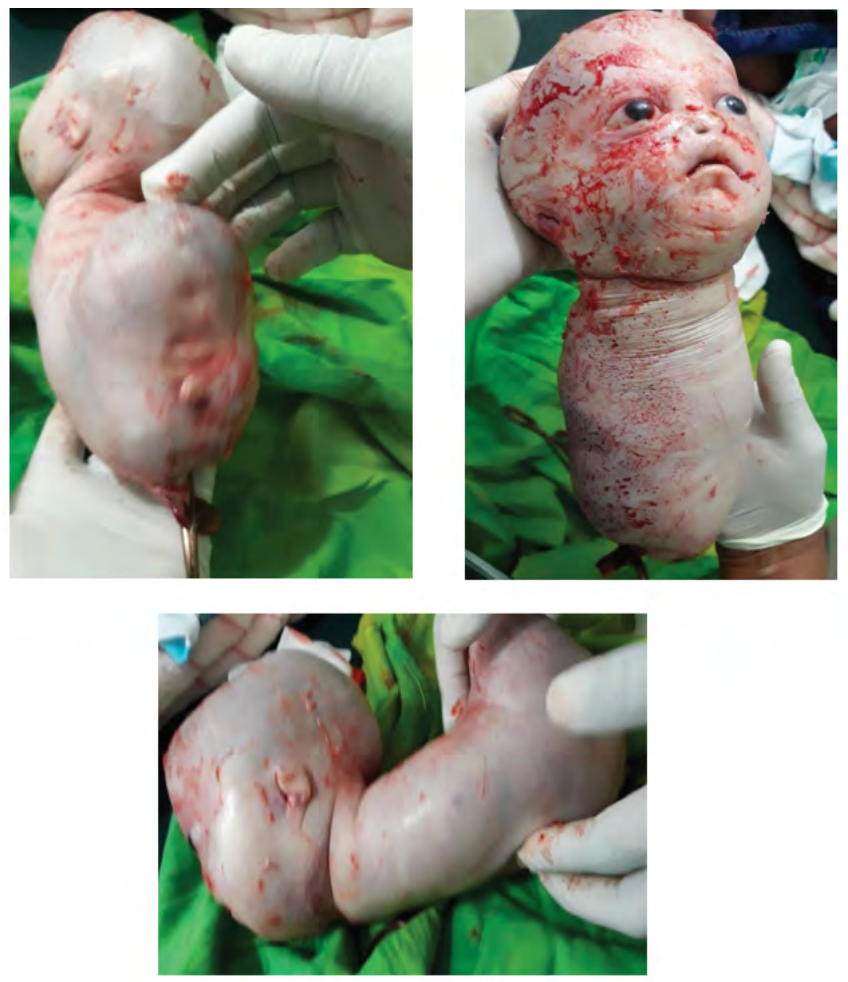

Figure 1 Case 1 New-born with tetra-amelia-1 syndrome (a) Caudal part of the new-born with a visible midline and a recess without anus or genitals. (b) Front view of the new-born showing a rudimentary nose, a mouth reduced to a slit, an absence of hair, eyelashes and eyebrows with a coloboma of the eyelids and a narrow chest. (c) Side view where we can see rudimentary ears.

with a coloboma of the eyelids, mild exophthalmos and aniridia. The mouth was alike an inverted $V$ without clear delimitation of the lips and the nose was rudimentary. The newborn was devoid of integuments (hair and eyebrows). The ears were reduced to sketches that looked like slits. The neck was short. The trunk was reduced to a $26 \mathrm{~cm}$ long conical structure with an umbilical cord at the bottom end. The chest was narrow. Just below the navel, the trunk widened backwards was present at the floor of the caudal pole, a midline with recessions and a budding which may correspond to a phallus of undetermined type. Agenesis of the pelvis, genitals and anal imperforation were noted. Fetal pathology has not been performed. However, death within 10 minutes of delivery and the conical appearance of the chest may suggest lung abnormalities.

\section{Case 2}

The second case was a 22-year-old primigravida referred to our facility for ultrasound scan at 37 weeks of gestation. She was not in a consanguineous marriage. She had tested negative for hepatitis B, HIV and syphilis. She was not tested for Toxoplasmosis and rubella. No ultrasound monitoring was done during her pregnancy. The clinical examination was consistent with fetal growth retardation (Fundal height: $26 \mathrm{~cm}$ ). Ultrasound findings showed the humerus to be distorted measuring $23.9 \mathrm{~mm}$ corresponding to 17 weeks of gestation. There was agenesis of the femur. The iliac wings were visible on ultrasound. No lung or heart abnormalities were identified. Delivery was initiated. The newborn had a female phenotype with an Apgar score of 9 at $5^{\text {th }}$ minute. The morphology of the head and the trunk was without particularity. The upper limbs were reduced to two $3 \mathrm{~cm}$-long stumps. Complete agenesis of the 2 lower limbs was noted. It was a symmetrical anomaly (Figure $\mathbf{2}$ ).

\section{Discussion}

Bermejo-Sanchez et al. described in 2011 the epidemiology of congenital amelia using data collected from 20 congenital anomalies surveillance programs, from all continents except Africa, between 1968 and 2006. In total, 326 cases of amelia were identified among 23,110,591 live births, stillbirths and abortions. The prevalence was 1.41/100,000 [2].

Tetra-amelia refers to as the complete absence of the limbs and occurs more rarely. To our knowledge, tetra-amelia- 1 is described in 7 families. It appears to follow an autosomal recessive inheritance. In all families, tetra-amelia-1 was associated with severe malformations of the other parts of the body including the face and head, anomalies of the nervous system, skeleton, and genitalia. The lungs were underdeveloped in many cases, which makes breathing difficult or impossible [3-6]. Zimmer et

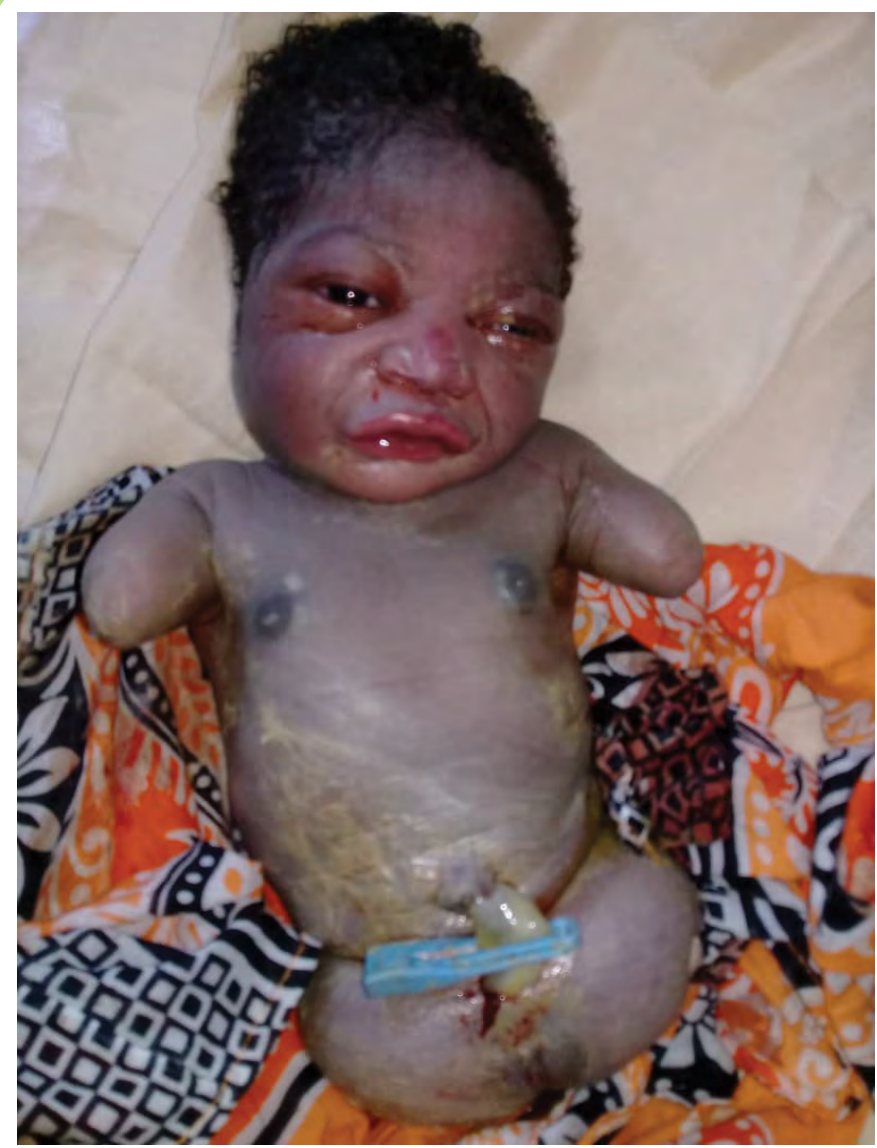

Figure 2 Case 2 Tetra-amelia-2 with symmetrical agenesis of the limbs. 
al. reported in 1985 a strongly inbred family in which 6 infants had tetra-amelia-1 and hydrocephalus. They described in one of the fetuses a total absence of pelvic bone, cleft lip and palate, arrhinia and aplasia of the ears. A bilateral left lung, a persistent arterial canal, an anal imperforation were also noted. Fetal testing eliminated the diagnosis of Robert's syndrome [4]. Other cases retrieved in the literature include that of Kosaki et al., in 1996, with a fetus of karyotype $46, \mathrm{XX}$ with tetraphocomelia and severe pulmonary hypoplasia in addition to face and head anomalies [7]. Rosenak et al. described a case of tetra-amelia with severe pulmonary hypoplasia in two fetuses of a non-consanguineous couple. Fetal testing ruled out diagnosis of Robert's syndrome [5]. Two additional cases were reported by Zlotogora et al. in 1993. Both patients died soon after birth and the authors suggested the existence of pulmonary hypoplasia. Niemann et al. reported a consanguineous Turkish family in which 4 of the 8 brothers suffered from tetra-amelia. In addition to the absence of the 4 limbs, the fetal examinations of 3 fetuses revealed multiple anomalies: cleft lips and /or palatine, laparoschisis, pulmonary anomalies, hypoplasia of the pelvis, atresia of the choanas, vagina and anal imperforation [8]. Finally, in 2005, Krahn et al. described 2 brothers born to inbred parents suffering from tetraamelia and severe pulmonary hypoplasia. The collarbones and shoulder blades were normal in the second fetus. The karyotype was normal [9].

Tetra-amelia-1 syndrome or TETAMS1 is caused by a homozygous mutation in the WNT3 gene on chromosome 17q21 with an autosomal recessive inheritance. Tetraamelia-2 syndrome (TETAMS2) is characterized by rudimentary limbs or a complete absence of the limbs, generally symmetrical as well as bilateral agenesis of the lungs in some cases. Are also usual anomalies of the pulmonary vascular system and dysmorphies including bilateral cleft lip and palate, ankyloglossia, mandibular hypoplasia, microretrognathia and labioscrotal aplasia [10].

Szenker-Ravi, studying 4 families of tetra-amelia with agenesis or pulmonary hypoplasia, noted a phenotypic heterogeneity with limb anomalies of varying severity [10]. Exome sequencing

\section{References}

1 Wilcox WR, Coulter CP, Schmitz ML (2015) Congenital limb deficiency disorders. Clin Perinatol 42: 281-300.

2 Bermejo SE, Cuevas L, Amar E (2011) Amelia: A multi-center descriptive epidemiologic study in a large dataset from the International Clearinghouse for Birth Defects Surveillance and Research, and overview of the literature. Am J Med Genet C Semin Med Genet 157: 288-304.

3 Zlotogora JSM, Shabany YO, Jarallah RY (1993) Syndrome of tetraamelia with pulmonary hypoplasia. Am J Med Genet 47: 570-571.

4 Zimmer EZ (1985) Tetra-amelia with multiple malformations in six male fetuses in one kindred. Europ. J Pediat 144: 412-414. in these 4 families has made it possible to identify truncating homozygous mutations in the RSPO2 gene [10]. Tetraamelia-2 syndrome is caused by a homozygous mutation in the RSPO2 gene (610575) located on chromosome 8q23 [10].

The phenotype of the first case described in this article corresponds to a tetra-amelia-1 syndrome due in particular to the presence of hydrocephalus, anomalies genitalia and a rudimentary nose. The narrow chest and early death before the 10th minute of life suggest severe pulmonary hypoplasia. This case highlights the phenotypic heterogeneity with an eyelid coloboma, hypertelorism, exophthalmos and rare appendages.

We consider the second case in our study to be a tetramelia-2 syndrome considering the symmetrical tetra-amelia with the presence of upper limb stumps. Diagnosis of tetra-amelia should be done early during ultrasound monitoring. Therefore, awareness should be raised about the importance of ultrasound monitoring and the use of $3 \mathrm{D} / 4 \mathrm{D}$ to improve the screening results. The diagnosis of a pelvis mass on ultrasound paired with amelia should raise suspicion for spleno-gonadal fusion limb defect syndrome.

In addition, the fetal examination and fetal testing using the evolving technologies of chromosomal microarray and exome and genome sequencing are to be encouraged in our settings. A better characterization of the cases makes it possible to provide advice to couples and a better knowledge of these clinical anomalies.

\section{Conclusion}

Tetra-amelia syndrome is scarce and gray areas still remain. These two cases, compared to what is already described in the literature, illustrate the phenotypic heterogeneity of tetraamelia. Given the rare incidence of these anomalies, it would be important to create an international registry of anomalies in order to report cases and set up a sample bank for extended genetic studies to parents.

5 Rosenak D (1991) Recurrent tetraamelia and pulmonary hypoplasia with multiple malformations in sibs. Am J Med Genet 38: 25-28.

6 Gershoni BR (1990) Roberts syndrome or "X-linked amelia" ?. Am J Med Genet 37: 569-572.

7 Kosaki K, Jones MC, Stayboldt C (1996) Zimmer phocomelia: delineation by principal coordinate analysis. Am J Med Genet 66: 55-59.

8 Niemann S(2004) Homozygous WNT3 mutation causes tetra-amelia in a large consanguineous family. Am J Hum Genet 74: 558-563.

9 Krahn M (2005)Tetra-amelia and lung aplasia syndrome: report of a new family and exclusion of candidate genes. Clin Genet 68: 558-560.

10 Szenker-RE, Altunoglu U (2018) RSPO2 inhibition of RNF43 and ZNRF3 governs limb development independently of LGR4/5/6. Nature 557: 564-569. 